\title{
Article \\ A Pore Network Approach to Study Throat Size Effect on the Permeability of Reconstructed Porous Media
}

\author{
Kai Xu ${ }^{1} \oplus$, Wei Wei ${ }^{2} \oplus$, Yin Chen ${ }^{2}$, Haitao Tian ${ }^{2}$, Sai $X u^{3 \oplus}$ and Jianchao Cai ${ }^{1, * \mathbb{C}}$ \\ 1 State Key Laboratory of Petroleum Resources and Prospecting, China University of Petroleum, \\ Beijing 102249, China; xuk@student.cup.edu.cn \\ 2 Institute of Geophysics and Geomatics, China University of Geosciences, Wuhan 430074, China; \\ weiwei@cug.edu.cn (W.W.); yinchen@cug.edu.cn (Y.C.); tht@cug.edu.cn (H.T.) \\ 3 School of Earth Resources, China University of Geosciences, Wuhan 430074, China; xus@cug.edu.cn \\ * Correspondence: caijc@cup.edu.cn
}

check for updates

Citation: Xu, K.; Wei, W.; Chen, Y.; Tian, H.; Xu, S.; Cai, J. A Pore Network Approach to Study Throat Size Effect on the Permeability of Reconstructed Porous Media. Water 2022, 14, 77. https://doi.org/ 10.3390/w14010077

Academic Editor: Giuseppe Pezzinga

Received: 17 November 2021

Accepted: 22 December 2021

Published: 3 January 2022

Publisher's Note: MDPI stays neutral with regard to jurisdictional claims in published maps and institutional affiliations.

Copyright: (C) 2022 by the authors. Licensee MDPI, Basel, Switzerland. This article is an open access article distributed under the terms and conditions of the Creative Commons Attribution (CC BY) license (https:// creativecommons.org/licenses/by/ $4.0 /)$.

\begin{abstract}
Permeability is usually considered to be related to porosity. However, rocks with the same porosity may have different permeabilities in some cases, because of the variations in pore and throat size and pore space connectivity. It is vitally important to understand the effect of throat size on the transport property. In this work, five sets of regular pore network models and six core-based models are employed to study the effect of throat size on permeability. Four kinds of random distributions, i.e., uniform, normal, Weibull, and log normal, are utilized to generate random pore size. Pore coordination number is set to be two and six for the verification of the effect of connectivity on permeability. Then, single-phase flow simulation is conducted based on the constructed pore network models. The simulation results show that permeability decreases significantly when only one of the nine throats reduces to half size in terms of diameter. The influence of pore coordination number on permeability is not obvious compared to that of small throat size. This study indicates that small throats play an extremely important role in determining permeability.
\end{abstract}

Keywords: pore network model; permeability; pore size distribution; pore coordination number; pressure distribution

\section{Introduction}

Micro-structure characterization of reservoir rock is the foundation in both basic flow mechanism studies and industrial applications. As emphasized by Torquato and $\mathrm{Lu}$ [1], the purpose of micro-structure characterization is to ascertain what is the essential morphological information, quantify it either theoretically or experimentally, and then employ the information to estimate the desired macroscopic properties of the heterogeneous materials. The well-known Kozeny-Carman (KC) equation is such a classical example of predicting permeability by finding the relationship between macroscopic property and microscopic morphological information [2]:

$$
K=\frac{\phi^{3} D_{p}^{2}}{c(1-\phi)^{2}}
$$

where $K$ is permeability, $\phi$ is porosity, $D_{p}$ is average diameter of sand grains, $c$ is the proportionality and unity factor. The equation holds for fluid flowing through packed beds with particle Reynolds numbers up to approximately 1.0. The KC equation is widely applied in permeability predictions.

However, it is not sufficient to characterize pore structure only using $D_{p}$, especially for tight sandstone, shale, and carbonate reservoirs, in which the pore size may vary from nanometers to larger than micrometers, and the variation of permeability is up to two or three orders of magnitude. For example, Purcell [3] showed that, in different 
sandstone samples, porosity varies from $16 \%$ to $25 \%$, while permeability varies from $23 \mathrm{mD}$ to $1150 \mathrm{mD}$. Nelson [4] found that each order of magnitude of change in throat size corresponds approximately to two orders of magnitude of change in permeability. Lai et al. [5] reported that some sandstones are with approximately equal porosity, but permeability varies from $0.01 \mathrm{mD}$ to $10 \mathrm{mD}$. Apparently, it is nearly impossible to accurately predict permeability by only one single theoretical equation, e.g., KC equation, even though many corrections are developed based on the original form [6-8]. In spite of this, the KC equation is still the most commonly used for permeability perdition because there are no better theoretical approaches.

Due to the development of computer hardware and various numerical simulation methods, it is practical to systematically investigate the effect of pore structure on permeability by numerical approaches. Golparvar et al. [9] presented a comprehensive review for various pore scale modeling methods, among which pore network model (PNM) is one of the easy-implemented choices for the study of fluid motion and transport. Up to date, PNM has been widely used in reactive transport [10], gas diffusion layer [11], and shale oil flow modeling [12]. The general idea of PNM is a mapping from the complex pore space continuum onto a regular or irregular lattice of sites and bonds [13]. The most remarkable advantage of PNM is the lower computational cost, which makes it possible to perform large scale modeling and repeated simulations. Fatt [14] designed four kinds of 2D networks with different coordinate numbers to simulate the displacement process through sandstone. Chatzis and Dullien [15] developed Fatt's PNM to 3D and simulated mercury intrusion in sandstones. Dong and Blunt [16] modified the maximal ball algorithm for PNM extraction from core samples, which is a common way for PNM extraction. Han [17] applied the shortest path algorithm in PNM to identify dominating flow paths in single-phase flow. Mahanta et al. [18] investigated PNM attributes in high-temperature heat-treated sandstones. Foroozesh et al. [19] studied stress-dependent fluid dynamics in shales using PNM. Mehmani et al. [20] summarized the development of PNM in the past 60 years, from which we found that PNM is commonly applied to study complex pore structures, e.g., extracting PNM from core samples or constructing stochastic PNM with specific porosity and pore coordination number (PCN). Only a few studies paid attention to the effect of small throat size on permeability. Generally, it is known that rocks with larger pore and throat size have larger permeability and lower pressure loss.

OpenPNM is an open source software package issued by Porous Materials Engineering and Analysis Lab at the University of Waterloo [21]. It supplies a framework for simulating single- and multi-phase transport process in porous materials. Many scholars have conducted their studies with OpenPNM. Tranter et al. [22] used OpenPNM to model water invasion and multiphase transport in compressed fuel cells. Schalenbach et al. [23] investigated the mechanism of gas permeability variation in nafion using OpenPNM. The above simulations agree well with experimental data. Yang et al. [12] performed pore-scale shale oil simulation using OpenPNM considering the effect of slippage and adsorption. Esteves et al. [24] implemented OpenPNM to study the geometry variation during dissolution process in carbonate rock. The above studies show the low computational-cost advantage of OpenPNM. In this study, OpenPNM is used to analyze the effect of small throat size on permeability by constructing PNM with different pore and throat sizes, pore coordination numbers, and porosity.

\section{Methods and Models}

\subsection{Permeability Calculation}

PNM method discretizes continuous pressure distribution onto pore locations. Then, the problem of flowing through porous media is transformed to the problem of flowing through connected pores and throats. The pressure at both ends of the throat are required 
for the calculation of flow rate. Based on the laminar flow equation, the mass conservation equation for each pore is expressed as:

$$
q_{i}=\sum_{j=1}^{n} g_{i j}\left(P_{j}-P_{i}\right)=0
$$

where $q_{i}$ is the net flow rate through pore $i$ (positive value means fluid flows into pore $i$, negative value means fluid flows out of pore $i), i$ denotes a certain pore, $j$ denotes all the pores neighboring to pore $i, n$ is the coordination number of pore $i, P_{i}$ is the pressure at pore $i, P_{j}$ is the pressure at each neighboring pore. For each pore, when the final steady state is achieved, the inlet and outlet flow rates are equal, which makes $q_{i}$ equal to 0 , except for the inlet pores and outlet pores. $g_{i j}$ is hydraulic conductivity for a conduit or a "half pore-throat-half pore" unit. Its value is determined as follows:

$$
\frac{1}{g_{i j}}=\frac{1}{g_{i}}+\frac{1}{g_{t, i j}}+\frac{1}{g_{j}}
$$

where $g_{i}, g_{j}$, and $g_{t, i j}$ are hydraulic conductivities for pore $i$, pore $j$, and the throat connecting pores $i$ and $j$, respectively. For each part of the conduit, the hydraulic conductivity is calculated by:

$$
g=\frac{A^{2}}{8 \pi \mu L}
$$

where $A$ is cross-section area, $\mu$ is fluid viscosity, $L$ is conduit length. For a pore, $A$ equals to its largest cross-section area, and $L$ equals to its radius approximately. For a throat, $A$ equals to its cross-section area, and $L$ equals to its length approximately. Figure 1 shows an example of a conduit. It should be noted that throat usually contributes to most parts of the hydraulic resistance for each conduit due to its smaller diameters.

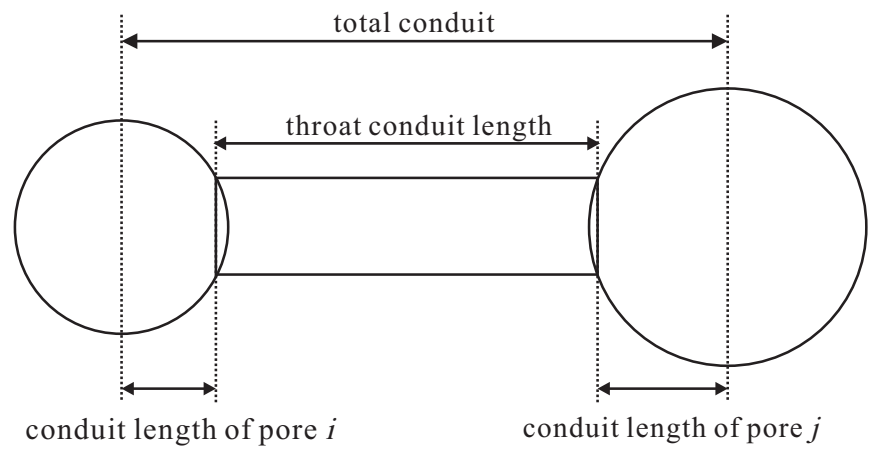

Figure 1. A conduit. The conduit lengths of two pores are a little smaller than their radii (Re-produced from Mehrez Agnaou's original work, for more information, ref to https: / github.com/PMEAL/ OpenPNM/issues/923 (accessed on 20 December 2021)).

Equation (2) only considers the situation of one pore and its connected pores. To extend it to the whole PNM, we obtain:

$$
Q=\sum_{i=1}^{N} q_{i}=\sum_{i=1}^{N} \sum_{j=1}^{n} g_{i j}\left(P_{j}-P_{i}\right)=0
$$

where $Q$ denotes the total flow rate through the whole PNM, $i$ denotes the index of all pores in the PNM. Considering all the pores in the PNM, the total flow rate at inlet pores equals to that at outlet pores (absolute values) at the final steady state. Solving Equation (5), the 
pressure distribution within all pores is obtained. Then the flow rate at inlet pores $\left(Q_{i n}\right)$ and permeability $(K)$ are calculated as follows:

$$
\begin{aligned}
& Q_{i n}=\sum_{i=1}^{N_{i n}} q_{i}=\sum_{i=1}^{N_{i n}} \sum_{j=1}^{n} g_{i j}\left(P_{j}-P_{i}\right) \\
& K=\frac{Q_{i n} \mu L_{s}}{S \Delta P}
\end{aligned}
$$

where $N_{\text {in }}$ denotes the index of the inlet pores, $S$ is the flow cross-section area of the PNM, $L_{S}$ is the vertical distance from inlet plane to outlet plane, $\Delta P$ is the applied pressure difference at two ends.

\subsection{Model Construction}

Five sets of PNM are designed according to the pore number (PN), throat number (TN), pore diameter (PD), throat diameter (TD), and PCN. Table 1 summarizes the information for the five model sets. Except for model set 5, all other PNM consist of 1000 pores, as a $10^{-3} \times 10^{-3} \times 10^{-3} \mathrm{~m}^{3}$ cubic network. TN is 900 for unidirectional PNM (model sets 1 and 3 ) and 2700 for fully connected PNM (model sets 2 and 4). For all the PNM, TD should be less than, or at most equal to the diameter of its connected pores.

Table 1. Model design scheme.

\begin{tabular}{ccccc}
\hline Model Sets & PCN & PCN $_{\text {ave }}$ & PD $\left(\times \mathbf{1 0}^{-\mathbf{5}} \mathbf{~ m}\right)$ & PNM Quantity \\
\hline 1 & 2 & 0.9 & $2.5,5.0$ & $2+85$ \\
2 & 6 & 2.7 & $2.5,5.0$ & $2+4$ \\
3 & 2 & 0.9 & {$[2.5,5.0]^{*},[0.1,10.0]$} & $24 \times 100$ \\
4 & 6 & 0.7 & {$[2.5,5.0],[0.1,10.0]$} & $24 \times 100$ \\
5 & & Extracted from core samples & 6
\end{tabular}

Note: ${ }^{*}[a, b]$ means that PD is random numbers within a and b. $\mathrm{PCN}_{\text {ave }}$ is calculated as TN/PN, which is usually smaller than PCN because of the boundary pores.

Model set 1 includes two blank control PNM and another 85 experimental PNM with various combinations of pore and throat diameters representing flow channels with different size. Figure 2a shows a unidirectional PNM belonging to model set 1, in which all pore diameters equal to $5.0 \times 10^{-5} \mathrm{~m}$, diameters of 300 throats equal to $2.5 \times 10^{-5} \mathrm{~m}$, and diameters of 600 throats equal to $5.0 \times 10^{-5} \mathrm{~m}$. Model set 2 consists of fully connected PNM, including two blank controls and another four experimental PNM, in which pore and throat diameters are set as $5.0 \times 10^{-5} \mathrm{~m}$ or $2.5 \times 10^{-5} \mathrm{~m}$.

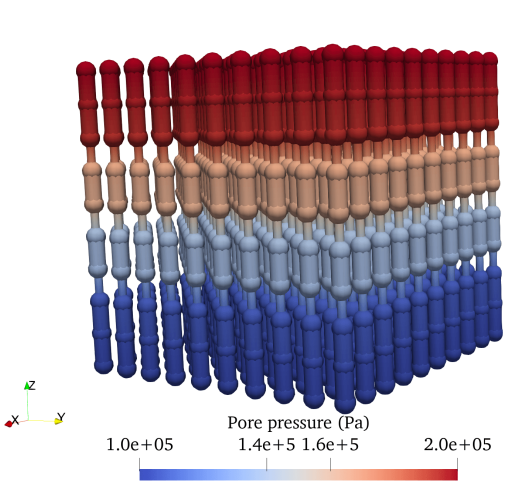

(a)

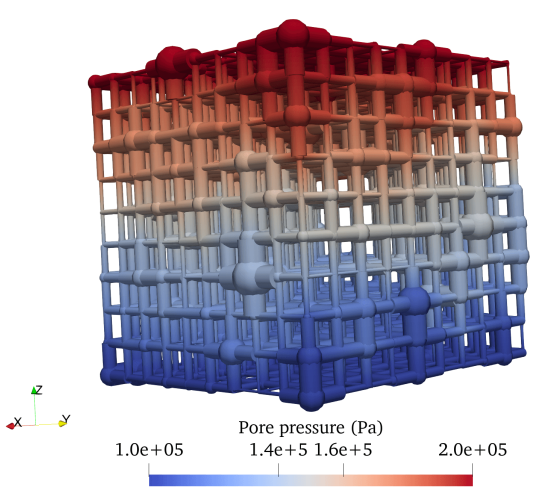

(b)

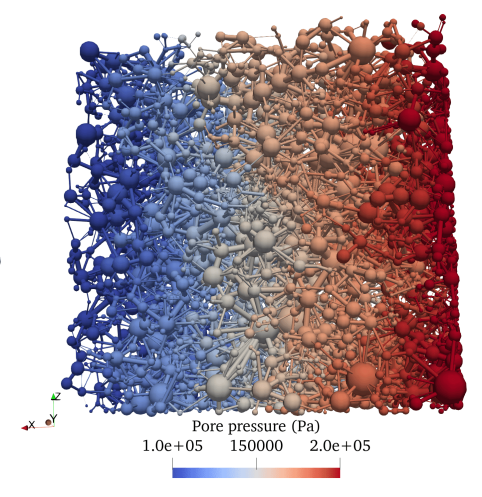

(c)

Figure 2. Three examples of PNM and the pressure distribution. (a) Unidirectional throat PNM. Colors show pressure distribution along the flow path. $\phi$ is $12.62 \%, K$ is $4.00 \times 10^{-12} \mathrm{~m}^{2}$. (b) Fully connected PNM. $\phi$ is $19.63 \%$. Pore, $K$ is $2.04 \times 10^{-12} \mathrm{~m}^{2}$. (c) Berea sandstone core samples. $\phi$ is $19.55 \%, K$ is $2.60 \times 10^{-12} \mathrm{~m}^{2}$. 
In model set 3, pore diameters are randomly generated according to 4 probability density functions (PDF), including uniform distribution, normal distribution, Weibull distribution, and log normal distribution. Figure 3 shows the four PDFs, among which Weibull and log normal distribution are two kinds of right-skewed distributions. Throat diameter is determined based on the diameters of its connected pores in three ways: $\min$, mean, and max. "min" means that throat diameter is calculated as the minimum of diameters of its connected pores and "mean" and "max" means average and maximum, respectively. $\mathrm{PCN}$ is the same as model set 1 . Besides, two pore diameter ranges are used for PNM design: $2.5 \times 10^{-5} \mathrm{~m}-5.0 \times 10^{-5} \mathrm{~m}$ and $1.0 \times 10^{-6} \mathrm{~m}-1.0 \times 10^{-4} \mathrm{~m}$. Combining four pore diameter generation schemes, three throat diameter generation schemes, and two pore diameter ranges, 24 basic PNM are obtained. In order to exclude chance factors embedded in the random pore diameter generation process, 100 repeated constructions are performed for each basic PNM. As a result, 2400 PNM are constructed. In model set 4, all the settings are the same as model set 3 , except that PCN of model set 4 is 6 . Figure $2 \mathrm{~b}$ shows a random fully connected PNM in model set 4 , in which pore diameters range from $1.0 \times 10^{-6} \mathrm{~m}$ to $1.0 \times 10^{-4} \mathrm{~m}$.
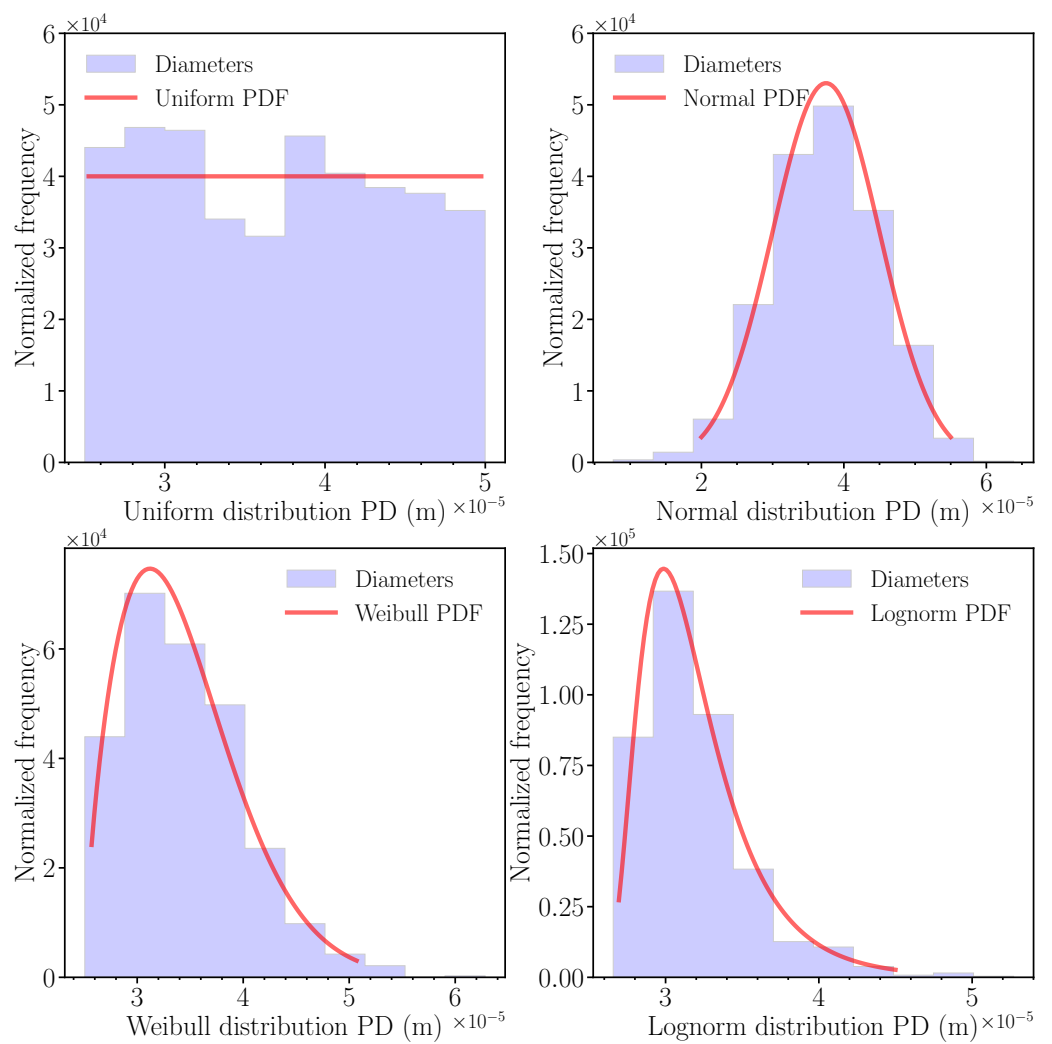

Figure 3. PDFs and the generation of pore diameters. Blue histogram represents the distribution of randomly generated 1000 pore diameter values.

In model set 5, six PNM are extracted from core samples, whose topological structures and pore and throat diameters are immutable. Figure 2c shows a PNM extracted from a Berea sandstone core sample, from which readers could find the complex structure in real rocks. This makes it nearly impossible to perform a precise control experiment only using core samples. That is the reason why all the above regular models are designed. More information about core sample PNM is shown in Section 3.5.

\subsection{Simulation Scheme}

In the simulation procedure, OpenPNM is used to: (1) construct PNM with varying pore and throat sizes, pore coordination numbers, and porosity; (2) import PNM extracted from X-ray computerized tomography (CT); (3) simulate single-phase incompressible 
laminar flow through the constructed PNM for the comparison of permeability and the evaluation of throat size effects.

The fluid used in the simulation is the built-in water in OpenPNM, which could be treated as single phase incompressible laminar flow. In model sets 1 to 4 , the pressure is applied in $z$-axis. In model set 5 , the pressure is applied in $x$-axis. The inlet and outlet pressures are 20,000 $\mathrm{Pa}$ and 10,000 $\mathrm{Pa}$, respectively, for all the five model sets. Model set 1 is employed to calculate permeability for different combinations of pore and throat diameters. Then, the relationship between permeability and number of small throats is acquired. Model set 2 is employed to calculate permeability and inlet flow rate distribution for the effect of connectivity. In model sets 3 and 4, simulation is performed on the 4800 random PNM to obtain the distributions of porosity and permeability. Porosity is also fixed within the range of $10-15 \%$ to study the effect of pore size distribution (PSD) on permeability. In model set 5, flow simulation is performed on core-sample PNM. Permeability is then calculated and compared to experimental results.

\section{Results and Discussion}

\subsection{Blank Control Group}

As mentioned in Section 2.3, the blank control group consists of four PNM. Pressure distribution, inlet flow rate, and permeability are obtained from the simulation results. It should be noted that half of each boundary pore is not considered for the calculation of pressure distribution because of the boundary effects, which means that the actual flow domain length is $9 \times 10^{-4} \mathrm{~m}$. Figure 4 a shows the cross-section view of the two unidirectional control PNM. Figure $4 \mathrm{~b}$ shows the pressure distribution within the four PNM.

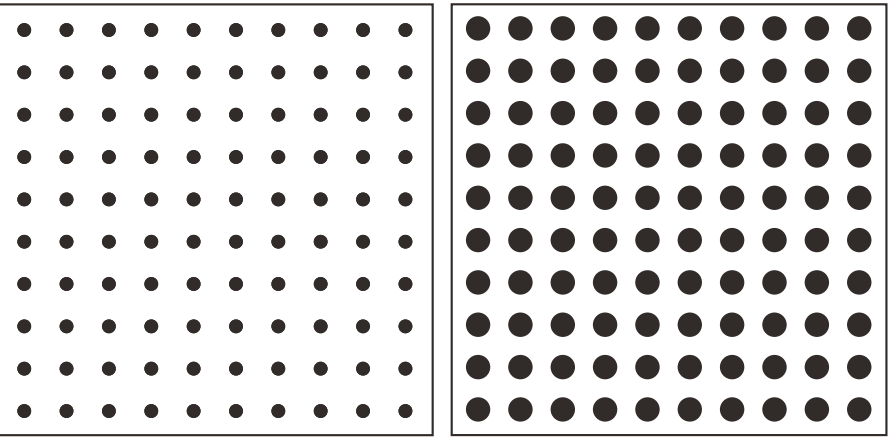

(a)

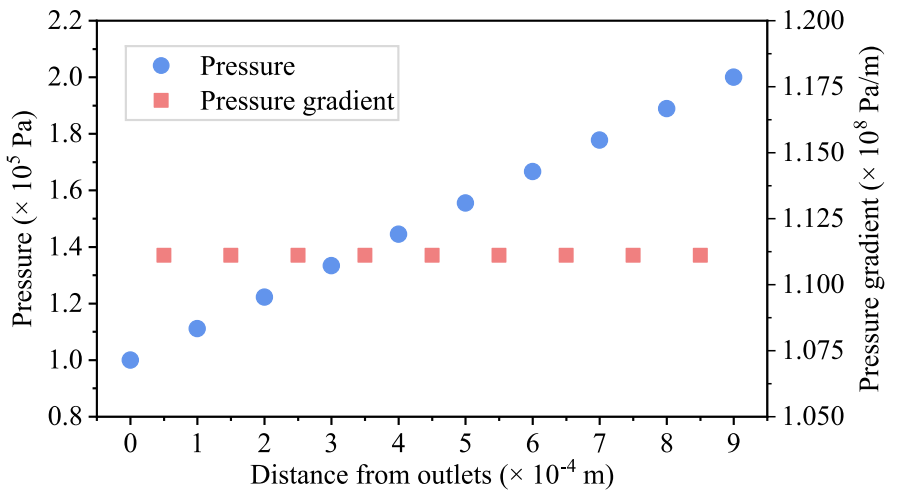

(b)

Figure 4. Cross-section view and pressure distribution of the four blank control PNM. (a) cross-section view of the two unidirectional blank control PNM. Left: pore diameters are $2.5 \times 10^{-5} \mathrm{~m}$; right: pore diameters are $5 \times 10^{-5} \mathrm{~m}$. (b) Pressure distribution of the four blank control PNM. Left $y$-axis represents pressure at the ten pores along one path (blue circles). Right $y$-axis represents pressure gradient at the nine throats along the same flow path (red squares). 
Though pore and throat diameters and pore coordination numbers are distinct among the four blank control PNM, they show the same pressure distribution (Figure 4b). That is because the constant pore and throat size doesn't produce extra resistance for fluid. As a result, the four situations all lead to linear pressure drop along the flow direction. From Table 2, it could be found that inlet flow rate and permeability are identical for PNM with the same pore and throat diameters, but are irrelevant to PCN, which seems impossible. But if readers recall that the simulation only presents the final steady-state condition, the result is reasonable. Besides, the simulation results are also compared to theoretical results calculated by the Hagen-Poiseuille equation, which shows the validity of the PNM method. One thing that should be noted is that the simulation result is always larger than the theoretical one, though the relative error is less than one thousandth.

Table 2. Simulation and theoretical comparison for blank control group.

\begin{tabular}{|c|c|c|c|c|c|c|c|c|}
\hline $\begin{array}{l}\text { Blank } \\
\text { Controls }\end{array}$ & $\begin{array}{l}\text { Model } \\
\text { Sets }\end{array}$ & $\phi(\%)$ & $\begin{array}{l}\text { Simulated } Q_{i n} \\
\left(\times 10^{-7} \mathrm{~m}^{3} / \mathrm{s}\right)\end{array}$ & $\begin{array}{c}\text { Theoretical } Q_{i n} \\
\left(\times 10^{-7} \mathrm{~m}^{3} / \mathrm{s}\right)\end{array}$ & $\begin{array}{l}\text { Relative Error } \\
\qquad\left(\times 10^{-4}\right)\end{array}$ & $\begin{array}{l}\text { Simulated } K \\
\left(\times 10^{-13} \mathrm{~m}^{2}\right)\end{array}$ & $\begin{array}{l}\text { Theoretical } K \\
\left(\times 10^{-13} \mathrm{~m}^{2}\right)\end{array}$ & $\begin{array}{l}\text { Relative Error } \\
\qquad\left(\times 10^{-4}\right)\end{array}$ \\
\hline 1 & 1 & 4.91 & 1.19265 & 1.19205 & 5.03 & 9.58738 & 9.58252 & 5.07 \\
\hline 2 & 1 & 19.63 & 19.0824 & 19.0728 & 5.03 & 153.398 & 153.320 & 5.09 \\
\hline 3 & 2 & 13.25 & 1.19265 & - & - & 9.58738 & - & - \\
\hline 4 & 2 & 53.01 & 19.0824 & - & - & 153.398 & - & - \\
\hline
\end{tabular}

\subsection{The Effect of Small Throats}

In model set 1 , flow simulations are conducted on the 85 unidirectional PNM. The results show variations of permeability and pressure distribution according to the configuration of small (large) pores and throats (shown in Figure 5).

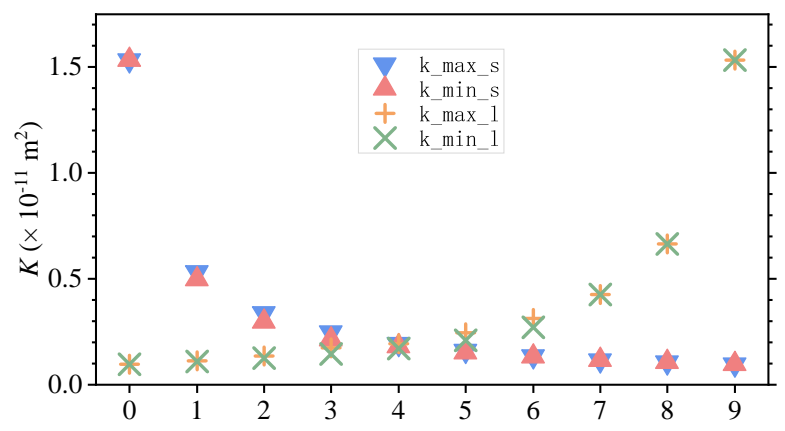

(a) The number of small (large) throats

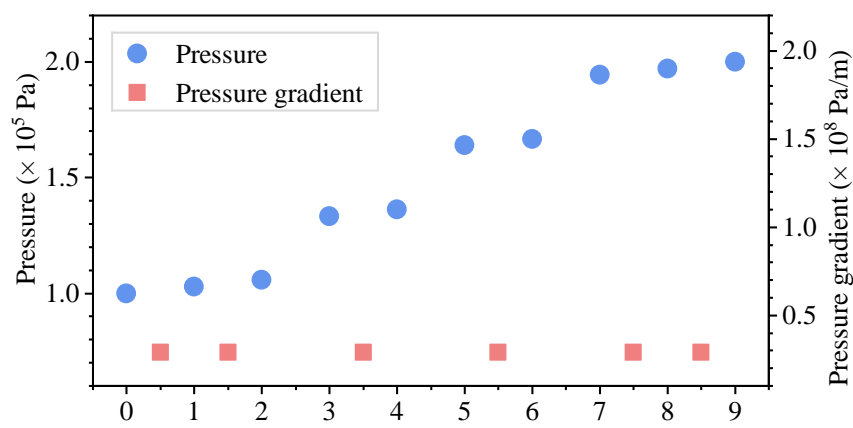

(c) Distance from outlets $\left(\times 10^{-4} \mathrm{~m}\right)$

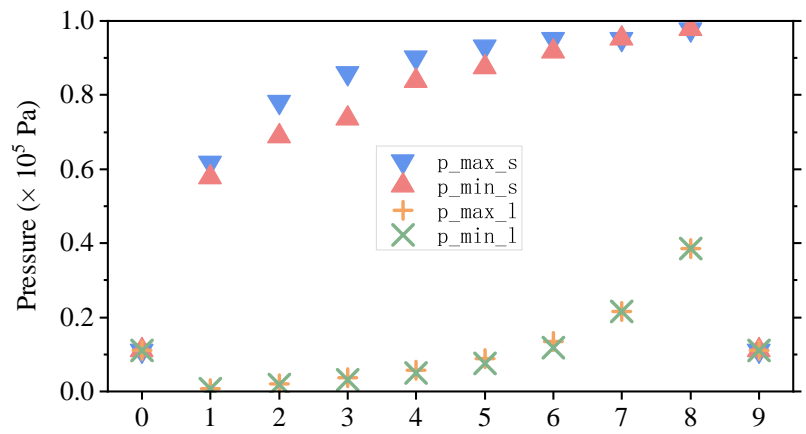

(b) The number of small (large) throats

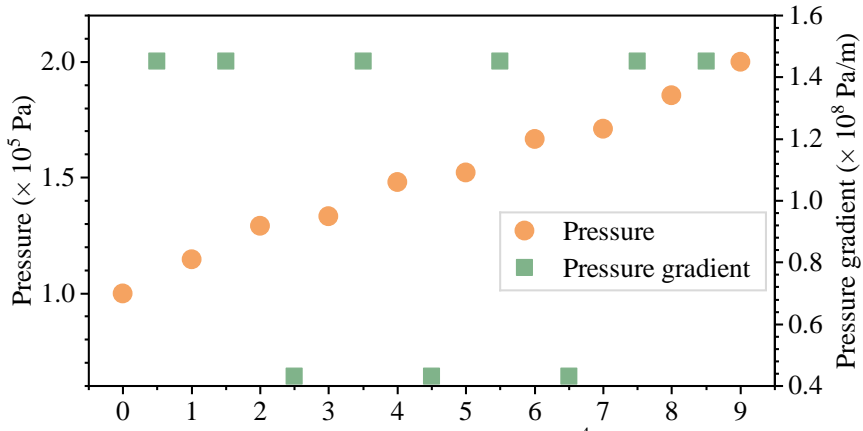

(d) Distance from outlets $\left(\times 10^{-4} \mathrm{~m}\right)$

Figure 5. Variations of permeability and pressure distribution according to the configuration of small (large) pores and throats. (a,b) variations of permeability and pressure according to the number of small (large) throats. (c,d) pressure distribution along flow direction within the PNM shown in Figure $2 \mathrm{a}$ and its contrary PNM in terms of pore diameters.

Figure 5 a shows that permeability decreases by nearly $65 \%$ when reducing the diameter of one throat to half, and permeability decreases by nearly $90 \%$ when reducing the 
diameter of five throats to half. However, the reverse operation (doubling the diameter of one throat) has no obvious effect on the improvement of the three flow properties. When doubling the diameter of four throats, permeability doubles. The above results reveal the restrictive effect of small throats on permeability, while the existence of a large throat surrounded by other small throats seems useless for the improvement of $K$. Figure $5 b$ shows the effect of throat size on the pressure distribution along a single flow channel, in which the first small throat takes up nearly $60 \%$ of the total pressure loss of the whole channel. Four small throats take up nearly $90 \%$ of the total pressure loss. Then, if all throats are equally small, the pressure is uniformly distributed along the channel again. However, one large throat surrounded by small ones takes only $0.8 \%$ of the total pressure loss, and eight large throats take up only $38 \%$ of the pressure loss. Figure $5 \mathrm{c}$ presents the pressure distribution within PNM shown in Figure 2a, which shows pressure decreases obviously when flowing through the small throats. As a contrast, Figure 5d illustrates the pressure distribution within PNM contrary to Figure 2a, which shows there is less pressure loss when flowing through large throats. All the above results confirm the restrictive effect of small throats on permeability. Besides, simulation results show that the position of small (large) throats have slight influence on permeability (see max and min values in Figure $5 a, b$ ), but it could be negligible compared to the effect of small (large) throat numbers.

\subsection{The Effect of Connectivity}

Intuitively, the increase of connectivity leads to increasing probability of forming highly conductive flow channels, which is the reason why better connectivity results in favorable permeability. Figure 6 presents a comparison of the pressure distribution difference among three PNM. In Figure 6a, the pressure distributes only in $z$-axis direction. Pressure difference vertical to $z$-axis is meaningless because no fluid motion happens in $x y$-plane. Figure 7a presents distributions of inlet flow rate for PNM shown in Figure 6a, in which two distinct areas with different inlet flow rate formed according to inlet pores' location. In Figure $6 \mathrm{~b}$, newly formed throats vertical to the $z$-axis produce effective pressure difference in all three directions, leading to more complex flow process and pressure distribution. In this case, local fluid motion in channels vertical to the $z$-axis may occur. As a result, the two distinct areas are replaced by a transition zone from max inlet flow rate to min inlet flow rate. Then, comparing inlet flow rate in the two PNM (Figure 7a,b), it could be found that max Inlet flow rate in Figure $7 \mathrm{a}$ is larger than that in Figure $7 \mathrm{~b}$, and the min inlet flow rate in Figure $7 \mathrm{a}$ is smaller than that in Figure $7 \mathrm{~b}$. Considering the concept of heterogeneity, it seems that the newly formed vertical-to-z-axis throats reduce the heterogeneity of PNM in Figure 6a. It should be noted that in this situation, when connectivity improves, porosity also becomes larger (from $18.18 \%$ to $49.48 \%$ ), while permeability only changes from $1.02 \times 10^{-11} \mathrm{~m}^{2}$ to $1.09 \times 10^{-11} \mathrm{~m}^{2}$, no more than $10 \%$ improvement. PNM in Figure $6 \mathrm{c}$ is contrary to that in Figure $6 \mathrm{~b}$ in terms of pore and throat size, and inlet flow rate distributions of the two PNM seem similar. However, considering the scale of color bars, readers could find that inlet flow rate of Figure $7 \mathrm{c}$ decreases to nearly $10 \%$ of that in Figure $7 \mathrm{~b}$, which is because there are more small throats in Figure 6c. According to the simulation results, it is found that connectivity has little effect on permeability, which is counter intuitive. One reason for this phenomenon is that the unidirectional PNM has already been well-connected, so any more extra improvement has no more obvious effect on permeability. 


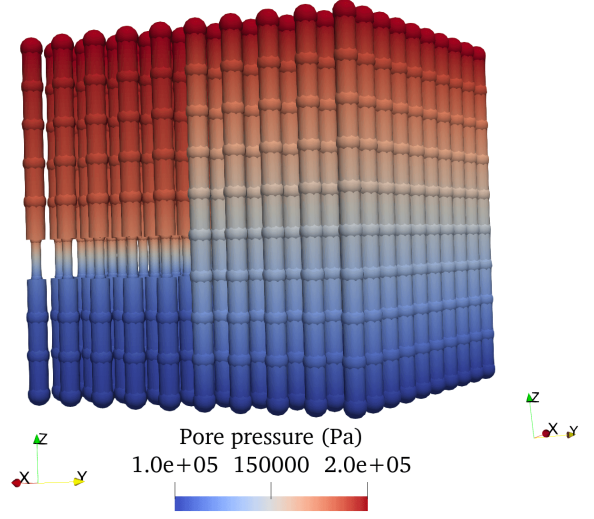

(a)

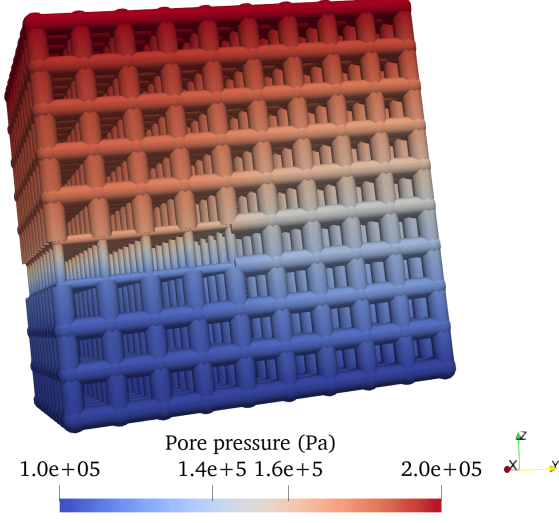

(b)

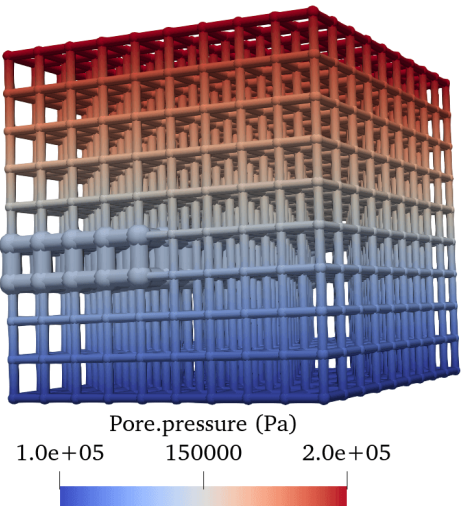

(c)

Figure 6. A comparison among three PNM for studying the effect of connectivity. (a) includes only unidirectional channels. (b) is fully connected. (c) is contrary to (b) in terms of pore and throat size. $\phi$ are $18.81 \%, 49.48 \%$, and $17.76 \%$, respectively. $K$ are $1.02 \times 10^{-11} \mathrm{~m}^{2}, 1.09 \times 10^{-11} \mathrm{~m}^{2}$, and $1.05 \times 10^{-12} \mathrm{~m}^{2}$, respectively. Color bar represents pressure distribution.

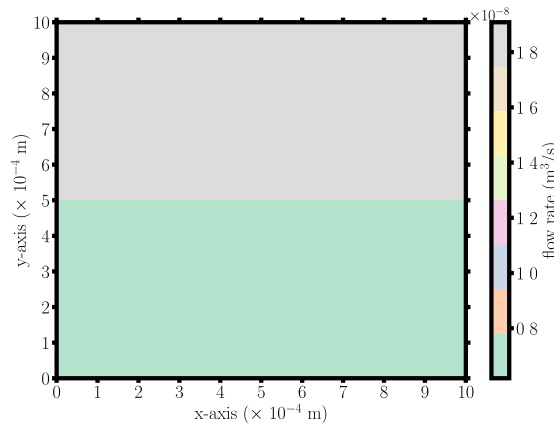

(a)

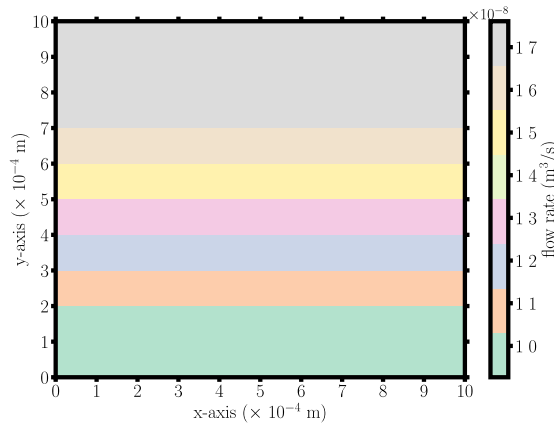

(b)

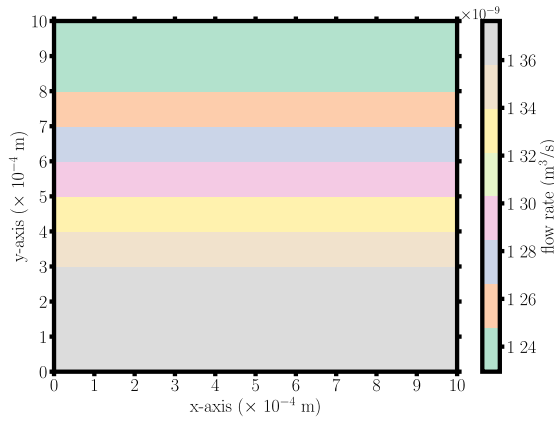

(c)

Figure 7. Sub figures $(\mathbf{a}-\mathbf{c})$ show inlet flow rate distributions in Figure 6a-c, respectively. Colors represent inlet flow rate $\left(\mathrm{m}^{3} / \mathrm{s}\right)$.

\subsection{Randomly Generated PNM}

According to the model design scheme in Section 2.2, there are 24 basic PNM in model set 3, which could be divided into two types. Type 1 includes 12 PNM, of which pore diameters are in the range of $2.5 \times 10^{-5} \mathrm{~m}-5.0 \times 10^{-5} \mathrm{~m}$. Type 2 includes another $12 \mathrm{PNM}$, of which pore diameters are in the range of $1.0 \times 10^{-6} \mathrm{~m}-1.0 \times 10^{-4} \mathrm{~m}$. Model set 4 also includes 24 basic PNM and could be divided into two types, same as model set 3 . After that, the 48 basic PNM are divided into two types according to range of pore diameters. Besides, porosity of type 2 is limited in the range of $10-15 \%$ by adjusting PDF parameters. Then, 100 repeated constructions and simulations are performed on each basic PNM to acquire the distributions of porosity and permeability. After this operation, chance factors are excluded as far as possible. Figure 8 shows the distributions of porosity and permeability for type 1 models. In Figure 8 a, porosity of unidirectional PNM varies from 5\% to $15 \%$, while porosity of fully connected PNM varies from $17 \%$ to $35 \%$. The dispersion degree of porosity for fully connected PNM is larger than that for unidirectional PNM, of which the reason is that the increasing throat number (from 900 to 2700) leads to increasing uncertainty of throat diameters and locations. Log normal and Weibull distributions usually produce smaller pores than normal and uniform distribution, which is the reason why the porosity of log normal and Weibull distributions is smaller than that of normal and uniform distributions. Normal distribution produces porosity with the largest dispersion degree, according to the box length in the horizontal axis. In Figure $8 b$, permeability varies from $1 \times 10^{-12} \mathrm{~m}^{2}$ to $1 \times 10^{-11} \mathrm{~m}^{2}$, and it does not increase proportionally as porosity increases, though they 
have an obvious positive relationship. For uniform distribution, permeability of fully connected PNM is $20 \%$ larger than that of unidirectional PNM. For three other distribution cases, the differences of permeability between unidirectional PNM and fully connected PNM are not apparent, which implies that permeability depends more on PSD than PCN.

Permeability of type 1 models is the result of the synthetic effect of porosity, PSD and PCN, so it is difficult to distinguish the contribution of throat size. Then, in type 2 models, pore diameter range is larger than that in type 1. Parameters of the four PDFs are also adjustable for the control of porosity. The average, first quartile, and third quartile of porosity are fixed in the range of $10-15 \%$. According to Figure $9 a$, this method is practicable. Equal porosity means that fully connected PNM should consist of smaller pores and throats compared to unidirectional PNM because the increasing throat number should be compensated by smaller throats for the conservation of $\phi$.

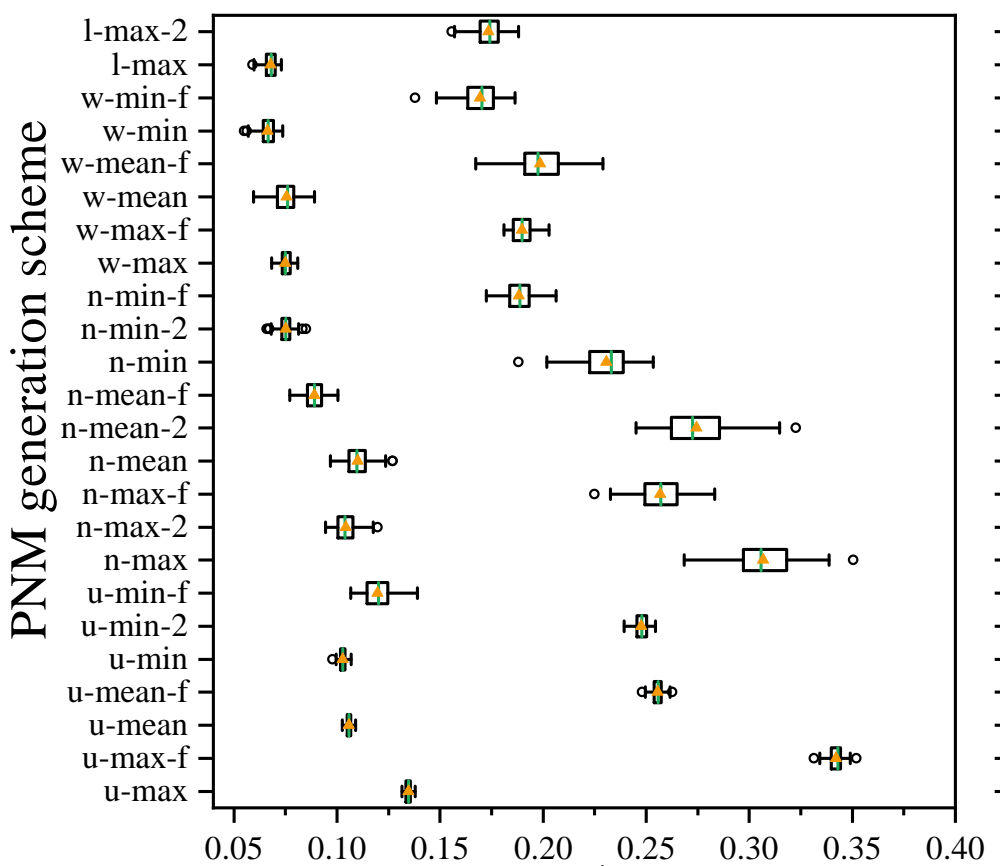

(a) $\phi$

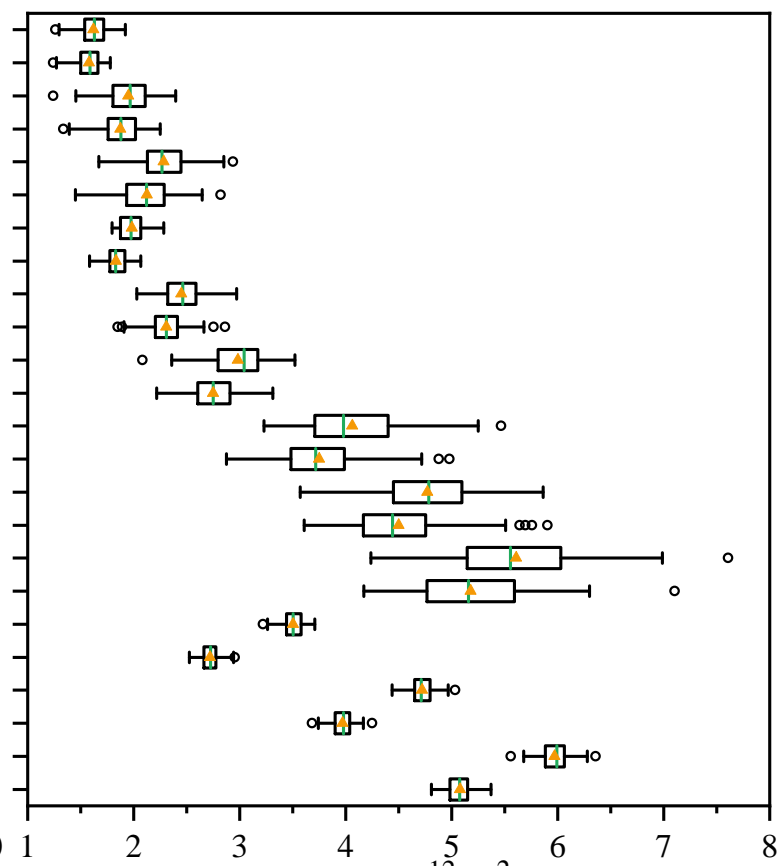

(b) $K\left(\times 10^{-12} \mathrm{~m}^{2}\right)$

Figure 8. Simulation results for type 1 models. (a) variation of $\phi,(\mathbf{b})$ variation of $K$ according to PNM generation scheme ( $y$-axis). The first letter of $y$-axis labels represents the PDF (" 1 " for log normal distribution; " $\mathrm{w}$ " for Weibull distribution; " $\mathrm{n}$ " for normal distribution; " $\mathrm{u}$ " for uniform distribution). The next three or four letters represent throat diameter generation scheme (min, mean, max, ref

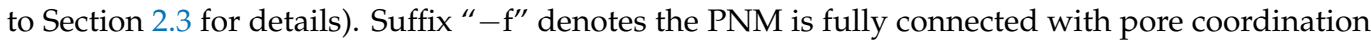
number of 6 .

In Figure 9b, permeability of log normal and Weibull distribution is generally smaller than that of normal and uniform distribution by 2 to 5 times. For the same pore and throat generation scheme, permeability of unidirectional PNM is larger than that of fully connected PNM by 2 to 10 times. For example, permeability decreases by nearly 4 times when PCN increases from 2 to 6 for uniform distribution PNM (u-mean and u-mean-f). In this case, increasing PCN leads to decreasing permeability, indicating that a large throat surpasses over a set of interconnected small throats in terms of flow capacity. PCN influences permeability by increasing the probability of finding a channel with high flow conductivity. The precondition of this probability is the existence of large pores and throats. In this sense, PSD is the fundamental factor affecting permeability. In type 2 models, it is the various pore diameter ranges that results in the different permeability. 


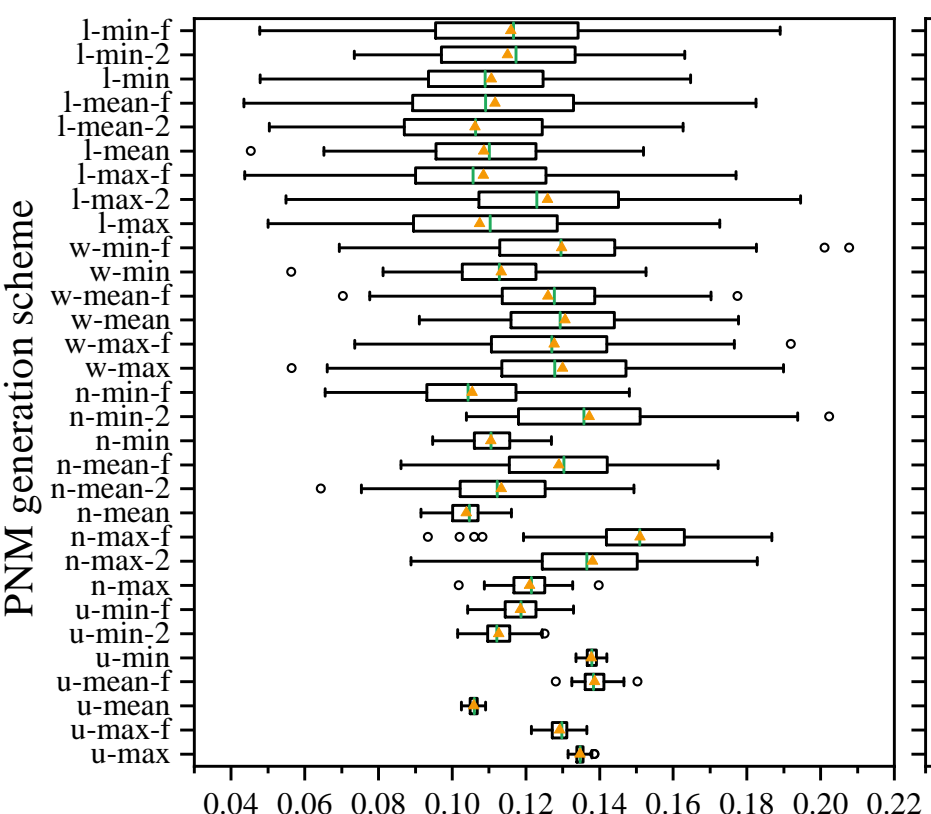

(a) $\phi$

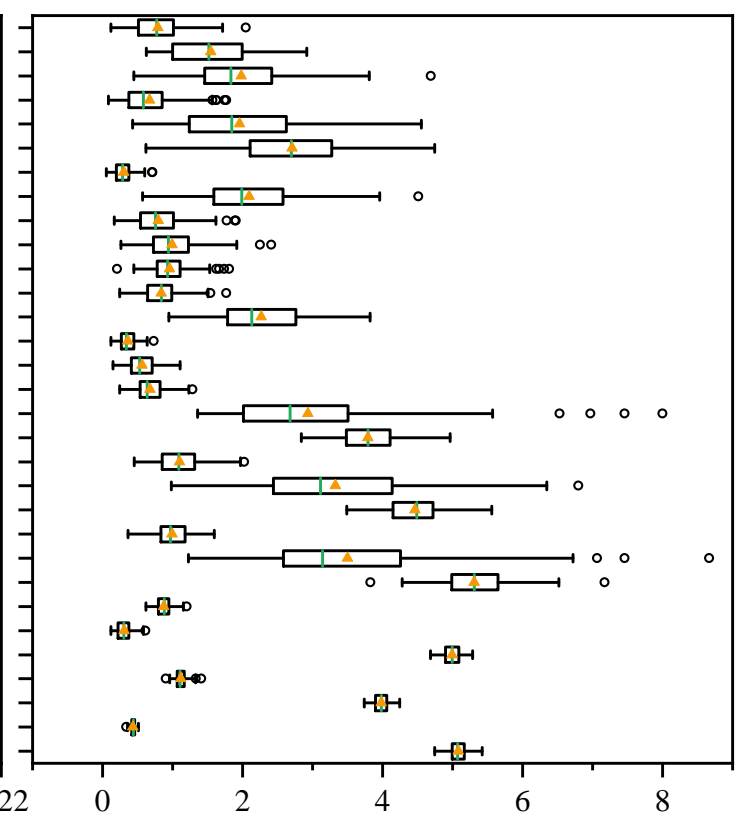

(b) $K\left(\times 10^{-12} \mathrm{~m}^{2}\right)$

Figure 9. Simulation results for type 2 models. (a) variation of $\phi,(\mathbf{b})$ variation of $K$ according to PNM generation scheme. Refer to Figure 8 for the explanation of figure notations. Suffix "-2" denotes derived PNM from the ones without the suffix by adjusting pore diameter ranges.

\subsection{PNM from Core Samples}

PNM and flow simulation results of the six core samples are shown in Table 3, including the data type, resolution, PSD, porosity, permeability, etc. The six PNM are downloaded from Imperial College London [16], including two sandstones, two sandpacks, and two carbonates. Network sizes are $2-3 \mathrm{~mm}$. The imported PNM should be trimmed before performing simulations to exclude disconnected clusters, isolated pores, and other useless elements.

Table 3. Core samples of model set 5 .

\begin{tabular}{|c|c|c|c|c|c|c|}
\hline PNM & Berea & S1 & F42A & LV60A & $\mathrm{C} 1$ & $\mathrm{C} 2$ \\
\hline Rock Type & \multicolumn{2}{|c|}{ Sandstone } & \multicolumn{2}{|c|}{ Sandpack } & \multicolumn{2}{|c|}{ Carbonate } \\
\hline Resolution $(\mu \mathrm{m})$ & 5.345 & 8.683 & 9.996 & 10.002 & 2.85 & 5.345 \\
\hline Size $(\mathrm{mm})$ & 2.14 & 2.60 & 3.00 & 3.00 & 1.14 & 2.14 \\
\hline $\mathrm{PN}$ & $6298(6004)$ & $1868(1717)$ & $1246(974)$ & 3135 (2636) & $4576(2612)$ & 8508 (4311) \\
\hline $\mathrm{TN}$ & 12098 (12067) & $2839(2824)$ & $2654(2651)$ & 7440 (7429) & $6700(5071)$ & $9818(7668)$ \\
\hline $\mathrm{PCN}_{\min }$ & $0(1)$ & $0(1)$ & $0(1)$ & $0(1)$ & $0(1)$ & $0(1)$ \\
\hline $\mathrm{PCN}_{\text {ave }}$ & $1.92(2.01)$ & $1.52(1.64)$ & $2.13(2.72)$ & $2.37(2.82)$ & $1.46(1.94)$ & $1.15(1.78)$ \\
\hline $\mathrm{PCN}_{\max }$ & 30 & 20 & 20 & 28 & 46 & 42 \\
\hline $\mathrm{PD}_{\min }(\mu \mathrm{m})$ & 4.5 & $1.8(2.0)$ & $2.4(13.1)$ & $2.1(8.3)$ & 0.58 & 1.075 (1.083) \\
\hline $\mathrm{PD}_{\text {ave }}(\mu \mathrm{m})$ & $30.7(31.5)$ & $51.2(53.9)$ & $89.3(109.0)$ & $70.9(80.2)$ & $14.1(17.7)$ & $22.8(28.3)$ \\
\hline $\mathrm{PD}_{\max }(\mu \mathrm{m})$ & 140.5 & 228.0 & 267.2 & 202.3 & 141.9 & 222.4 \\
\hline $\mathrm{TD}_{\min }(\mu \mathrm{m})$ & 1.1 & 1.7 & 20.0 & 20.0 & 0.58 & $1.20(1.28)$ \\
\hline $\mathrm{TD}_{\text {ave }}(\mu \mathrm{m})$ & $14.02(14.04)$ & $23.9(24.0)$ & $55.75(55.78)$ & $40.27(40.30)$ & $7.86(8.65)$ & $11.96(12.77)$ \\
\hline $\mathrm{TD}_{\max }(\mu \mathrm{m})$ & 80.1 & 104.2 & 162.1 & 126.6 & 63.6 & 103.5 \\
\hline$\phi(\%)$ & $19.62(19.55)$ & $14.12(14.05)$ & 32.85 (32.75) & $36.51(36.30)$ & $23.75(21.24)$ & $17.15(14.03)$ \\
\hline$\phi \exp (\%)$ & 19.6 & 14.1 & 33.0 & 37.7 & 23.3 & 16.8 \\
\hline$K\left(\times 10^{-12} \mathrm{~m}^{2}\right)$ & 2.600 & 3.589 & 198.12 & 73.05 & 0.292 & 0.047 \\
\hline$K_{\exp }\left(\times 10^{-12} \mathrm{~m}^{2}\right)$ & 1.360 & 1.969 & 61.0 & 27.2 & 0.785 & 0.038 \\
\hline
\end{tabular}

Notes: Values in the parentheses corresponds to the result of trimming. Subscript "exp" denotes the experimental results. 
After trimming, Berea sandstone has the largest pore and throat numbers. Sandpack F42A has the smallest pore and throat numbers. The two carbonates have the smallest $\mathrm{PCN}_{\text {ave }}$, but the largest $\mathrm{PCN}_{\max }$, which shows their high heterogeneity. Sandpacks have the largest pore and throat diameter distributions. The same rock types have similar PSD features. Comparing the simulation and experimental results, it could be found that the extracted PNM produce precise microstructures of core samples in terms of porosity. Sandpacks have the largest porosity. Permeability simulation results deviate from reference values by less than 3 times. As a comparison, Yang et al. [25] performed a pore to pore validation of PNM against a micro-model experiment, in which the relative error of PNM to micro-model experiment varied from 15 to $60 \%$. However, the amount of deviation did not disturb the evaluation of throat size effect on permeability because simulation and experimental results of the same rock type are in the same order of magnitude. Porosity of sandpacks is the largest, while that of carbonates is the smallest. According to the above analysis, it could be concluded that sandpacks have the largest porosity, PSD, and permeability, so it is hard to distinguish which factor contributes mostly to permeability for sandpacks. Sandstones and carbonates have similar porosity, but the PSD of carbonates is smaller than that of sandstones. And permeability of carbonates is smaller than that of sandstones. So, it may be reasonable to infer that small PSD limits carbonates' permeability, which shows the restrictive effect of small throat size on permeability in core sample PNM.

\section{Conclusions}

In this study, five sets of PNM with varying porosity, PSD, and PCN were constructed. Single phase flow simulation was performed on the generated PNM. Simulation results revealed the restrictive effect of small throat size on permeability. Most of the pressure loss was concentrated in the small parts of a channel. Increasing PCN could improve the connectivity of PNM and reduce its heterogeneity. However, it is not very effective for the improvement of permeability. Permeability decreased with the increase of $\mathrm{PCN}$, indicating that permeability depended more on throat size rather than PCN.

In addition, six PNM extracted from core samples were employed for the confirmation of the conclusions. The regular PNM generated in this study do not resemble structures in real rocks. This work may supply a guidance for porous structure design and permeability evaluation.

Author Contributions: Conceptualization, K.X. and W.W.; methodology, K.X. and Y.C.; software, K.X.; validation, H.T., S.X. and J.C.; formal analysis, W.W.; investigation, K.X.; resources, J.C.; data curation, J.C.; writing —original draft preparation, K.X.; writing—review and editing, W.W., Y.C. and S.X.; visualization, H.T.; supervision, J.C.; project administration, W.W.; funding acquisition, J.C. All authors have read and agreed to the published version of the manuscript.

Funding: This research was funded by the National Natural Science Foundation of China (No. 42172159).

Data Availability Statement: Berea sandstone PNM data presented in Section 3.5 is openly available in FigShare at https:// doi.org/10.6084/m9.figshare.1153794.v2; S1 sandstone PNM data presented in Section 3.5 is openly available in FigShare at https:// doi.org/10.6084/m9.figshare.1189274.v1; F42A sandpack PNM data presented in Section 3.5 is openly available in FigShare at https:// doi.org/10.6 084/m9.figshare.1189259.v1; LV60A sandpack PNM data presented in Section 3.5 is openly available in FigShare at https:/ / doi.org/10.6084/m9.figshare.1153795.v2; C1 carbonate PNM data presented in Section 3.5 is openly available in FigShare at https:/ / doi.org/10.6084/m9.figshare.1189257.v1; C2 carbonate PNM data presented in Section 3.5 is openly available in FigShare at https://doi.org/10.6 084/m9.figshare.1189258.v1p.

Acknowledgments: We acknowledge PMEAL for developing OpenPNM, and Imperial College London for the availability of core sample PNM. Version 2.1.0 is implemented in this research.

Conflicts of Interest: The authors declare that they have no known competing financial interests or personal relation relationships that could have appeared to influence the work reported in this paper. 


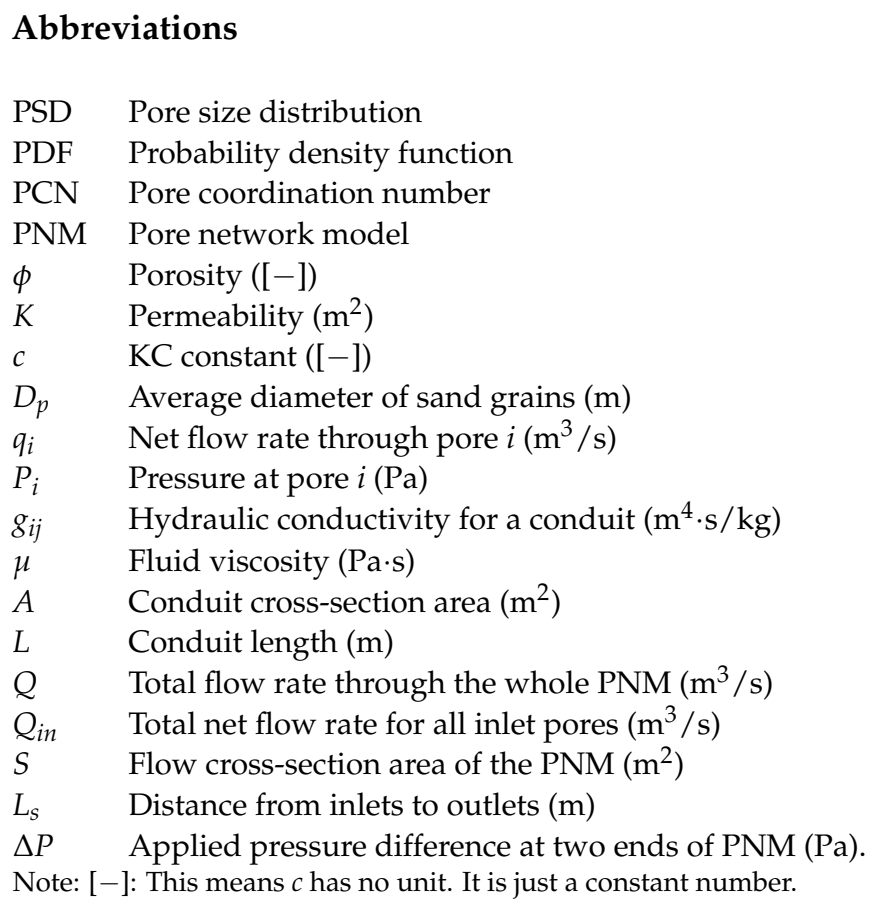

\section{References}

1. Torquato, S.; Lu, B. Chord-length distribution function for two-phase random media. Phys. Rev. E 1993, 47, 2950-2953. [CrossRef] [PubMed]

2. $\mathrm{Xu}, \mathrm{P} . ; \mathrm{Yu}, \mathrm{B}$. Developing a new form of permeability and Kozeny-Carman constant for homogeneous porous media by means of fractal geometry. Adv. Water Resour. 2008, 31, 74-81. [CrossRef]

3. Purcell, W.R. Capillary pressures-Their measurement using mercury and the calculation of permeability therefrom. J. Pet. Technol. 1949, 1, 39-48. [CrossRef]

4. Nelson, P.H. Pore-throat sizes in sandstones, tight sandstones, and shales. AAPG Bull. 2009, 93, 329-340. [CrossRef]

5. Lai, J.; Wang, G.; Wang, Z.; Chen, J.; Pang, X.; Wang, S.; Zhou, Z.; He, Z.; Qin, Z.; Fan, X. A review on pore structure characterization in tight sandstones. Earth-Sci. Rev. 2018, 177, 436-457. [CrossRef]

6. Mavko, G.; Nur, A. The effect of a percolation threshold in the Kozeny-Carman relation. Geophysics 1997, 62, 1480-1482. [CrossRef]

7. Costa, A. Permeability-porosity relationship: A reexamination of the Kozeny-Carman equation based on a fractal pore-space geometry assumption. Geophys. Res. Lett. 2006, 33, L02318. [CrossRef]

8. Wei, W.; Cai, J.; Xiao, J.; Meng, Q.; Xiao, B.; Han, Q. Kozeny-Carman constant of porous media: Insights from fractal-capillary imbibition theory. Fuel 2018, 234, 1373-1379. [CrossRef]

9. Golparvar, A.; Zhou, Y.; Wu, K.; Ma, J.; Yu, Z. A comprehensive review of pore scale modeling methodologies for multiphase flow in porous media. Adv. Geo-Energy Res. 2018, 2, 418-440. [CrossRef]

10. Raoof, A.; Nick, H.M.; Hassanizadeh, S.M.; Spiers, C.J. PoreFlow: A complex pore-network model for simulation of reactive transport in variably saturated porous media. Comput. Geosci. 2013, 61, 160-174. [CrossRef]

11. Qin, C. Water transport in the gas diffusion layer of a polymer electrolyte fuel cell: Dynamic pore-network modeling. J. Electrochem. Soc. 2015, 162, F1036-F1046. [CrossRef]

12. Yang, Y.; Wang, K.; Zhang, L.; Sun, H.; Zhang, K.; Ma, J. Pore-scale simulation of shale oil flow based on pore network model. Fuel 2019, 251, 683-692. [CrossRef]

13. Gostick, J.T.; Ioannidis, M.A.; Fowler, M.W.; Pritzker, M.D. Pore network modeling of fibrous gas diffusion layers for polymer electrolyte membrane fuel cells. J. Power Sources 2007, 173, 277-290. [CrossRef]

14. Fatt, I. The network model of porous media. Pet. Trans. AIME 1956, 207, 144-181. [CrossRef]

15. Chatzis, I.; Dullien, F.A.L. Modelling pore structure by 2-D and 3-D networks with applicationto sandstones. J. Can. Pet. Technol. 1977, 16, 97-108. [CrossRef]

16. Dong, H.; Blunt, M.J. Pore-network extraction from micro-computerized-tomography images. Phys. Rev. E 2009, 80, 036307. [CrossRef]

17. Han, J. The Application of the Shortest Path Algorithm in Pore Network Model. Master's Thesis, University of Electronic Science and Technology of China, Chengdu, China, 2019.

18. Mahanta, B.; Vishal, V.; Ranjith, P.G.; Singh, T.N. An insight into pore-network models of high-temperature heat-treated sandstones using computed tomography. J. Nat. Gas Sci. Eng. 2020, 77, 103227. [CrossRef]

19. Foroozesh, J.; Mohamed Abdalla, A.I.; Zivar, D.; Douraghinejad, J. Stress-dependent fluid dynamics of shale gas reservoirs: A pore network modeling approach. J. Nat. Gas Sci. Eng. 2021, 95, 104243. [CrossRef] 
20. Mehmani, A.; Verma, R.; Prodanović, M. Pore-scale modeling of carbonates. Mar. Pet. Geol. 2020, 114, 104141. [CrossRef]

21. Gostick, J.; Aghighi, M.; Hinebaugh, J.; Tranter, T.; Hoeh, M.A.; Day, H.; Spellacy, B.; Sharqawy, M.H.; Bazylak, A.; Burns, A.; et al. OpenPNM: A pore network modeling package. Comput. Sci. Eng. 2016, 18, 60-74. [CrossRef]

22. Tranter, T.G.; Gostick, J.T.; Burns, A.D.; Gale, W.F. Pore network modeling of compressed fuel cell components with OpenPNM. Fuel Cells 2016, 16, 504-515. [CrossRef]

23. Schalenbach, M.; Hoeh, M.A.; Gostick, J.T.; Lueke, W.; Stolten, D. Gas permeation through nafion. Part 2: Resistor network model. J. Phys. Chem. C 2015, 119, 25156-25169. [CrossRef]

24. Esteves, B.F.; Lage, P.L.; Couto, P.; Kovscek, A.R. Pore-network modeling of single-phase reactive transport and dissolution pattern evaluation. Adv. Water Resour. 2020, 145, 103741. [CrossRef]

25. Yang, J.; Bondino, I.; Regaieg, M.; Moncorgé, A. Pore to pore validation of pore network modelling against micromodel experiment results. Comput. Geosci. 2017, 21, 849-862. [CrossRef] 